\title{
Adaptive Guard Time for Energy-Efficient IEEE 802.15.4 TSCH Networks
}

Mavromatis, Alex; Papadopoulos, Georgios; Elsts, Atis; Montavont, Nicolas; Piechocki, Robert; Tryfonas, Theo; Oikonomou, George; Fafoutis, Xenofon

\section{Published in:}

Proceedings of 17th IFIP WG 6.2 International Conference on Wired/Wireless Internet Communications

Link to article, DOI:

10.1007/978-3-030-30523-9_2

Publication date:

2019

Document Version

Peer reviewed version

Link back to DTU Orbit

Citation (APA):

Mavromatis, A., Papadopoulos, G., Elsts, A., Montavont, N., Piechocki, R., Tryfonas, T., Oikonomou, G., \& Fafoutis, X. (2019). Adaptive Guard Time for Energy-Efficient IEEE 802.15.4 TSCH Networks. In Proceedings of 17th IFIP WG 6.2 International Conference on Wired/Wireless Internet Communications (pp. 15-26). Springer. Lecture Notes in Computer Science Vol. 11618 https://doi.org/10.1007/978-3-030-30523-9_2

\section{General rights}

Copyright and moral rights for the publications made accessible in the public portal are retained by the authors and/or other copyright owners and it is a condition of accessing publications that users recognise and abide by the legal requirements associated with these rights.

- Users may download and print one copy of any publication from the public portal for the purpose of private study or research.

- You may not further distribute the material or use it for any profit-making activity or commercial gain

- You may freely distribute the URL identifying the publication in the public portal 


\title{
Adaptive Guard Time for Energy-Efficient IEEE 802.15.4 TSCH Networks ${ }^{\star}$
}

\author{
Alex Mavromatis ${ }^{1}$, Georgios Z. Papadopoulos ${ }^{2}$, Atis Elsts ${ }^{3,1}$, \\ Nicolas Montavont ${ }^{2}$, Robert Piechocki ${ }^{1}$, Theo Tryfonas ${ }^{1}$, George Oikonomou ${ }^{1}$, \\ and Xenofon Fafoutis ${ }^{4}$ \\ 1 University of Bristol, Bristol, UK \\ \{alex.mavromatis,r.j.piechocki, theo.tryfonas, g.oikonomou\}@bristol.ac.uk \\ 2 IMT Atlantique, Rennes, France \\ \{georgios.papadopoulos, nicolas.montavont\}@imt-atlantique.fr \\ 3 Institute of Electronics and Computer Science (EDI), Riga, Latvia \\ atis.elsts@edi.lv \\ 4 Technical University of Denmark (DTU), Kgs. Lyngby, Denmark \\ xefa@dtu.dk
}

\begin{abstract}
Several Internet of Things (IoT) applications have strict performance requirements, in terms of reliability and power consumption. IEEE 802.15.4 Time Slotted Channel Hopping (TSCH) is a recently standardised Medium Access Control (MAC) protocol that supports these requirements by keeping the nodes time-synchronised. In order to ensure successful communication between a sender and a receiver, the latter starts listening shortly before the expected frame's arrival. This time offset is called guard time and it aims to reduce the probability of missed frames due to clock drift. This paper investigates the impact of the guard time on the energy consumption and proposes a scheme for the decentralised adaptation of the guard time in each node depending on its hopdistance from the sink. Simulations and test-bed experiments demonstrate that guard time adaptation can reduce the energy consumption by up to $50 \%$, without compromising the reliability of the network.
\end{abstract}

Keywords: IEEE 802.15.4 · TSCH · Internet of Things.

\section{Introduction}

The Internet of Things (IoT) consists of smart, uniquely identifiable and connected objects that construct a network of things suitable for wireless industrialtype of scenarios. Nowadays, industry is considering IoT technologies to accelerate the fourth industrial revolution, also called the Industry 4.0 [22]. It consists of applying the IoT automations to reduce the operational and management cost, to simplify the production chains, to ease the deployments and to allow for adaptability in the factories [13]. Applications such as e-health, cargo transportation, smart buildings, automotive industry or airport logistics, all share

* This work was partially supported by EPSRC, Grant EP/K031910/1. 
hard performance requirements, such as ultra-low energy consumption, latency and jitter, as well as high network reliability. Yet, the currently available IoT technologies straggle to provide such services, since they are extremely costly in terms of energy consumption. Therefore, in order to realise the vision of the IoT, the current standards must consider energy-efficient algorithms and solutions within the functionality of the wireless infrastructure to provide stable and predictable performance. This study focuses on reducing the energy consumption, without compromising the Packet Delivery Ratio (PDR).

The IEEE 802.15.4-2015 standard [2], published in 2016, aims to offer a certain quality of service for applications with an industrial-level of performance requirements. Among the Medium Access Control (MAC) protocols defined in this standard, Time Slotted Channel Hopping (TSCH) is designed for low-power and reliable networking in Low-power Lossy Networks (LLNs). In TSCH, the nodes in the network must remain synchronised throughout the deployment lifetime. Indeed, the nodes maintain a level of synchronisation by periodically compensating for the clock drifts. To avoid the loss of synchronisation between the synchronisation events, a TSCH receiver wakes up before the scheduled transmission by a fixed period of time. This period of time is called guard time and is responsible for significant energy wastage in idle listening. In previous work $[15,17,18]$, we highlighted the effect of guard time on a TSCH network and identified that, when employing the $6 \mathrm{TiSCH}$ minimal schedule, most of the energy consumed is wasted in idle listening.

Unlike other types of synchronous sensor networks [3], in TSCH the guard time is typically configured statically and, therefore, needs to account for the worst case scenario, not only in terms of clock drift, but also in terms of network size. Instead, this work demonstrates that TSCH could operate more energyefficiently by adapting the guard time in a decentralised manner. To this aim, this paper first proposes a decentralised heuristic algorithm for guard time adaptation in multi-hop TSCH networks, implemented for Contiki OS. The performance evaluation, which includes simulations and test-bed experiments, demonstrates that adapting the guard time on each node can significantly improve the energy consumption of TSCH networks (i.e., by up to $50 \%$ ) without compromising their reliability.

To summarise, this paper extends our earlier works $[15,17,18]$ with the following contributions. Firstly, different to our preliminary works, we evaluate and quantify the benefits of guard time adaptation in multi-hop line topologies and random multi-hop topologies and under various application-layer traffic conditions via a series of COOJA (a simulator for Contiki OS) simulations. Secondly, we implement and experimentally evaluate the proposed algorithm in test-bed experiments, conducted in the SPHERE house test-bed [8], a deployment of CC2650-based sensor nodes [10] in a real residential environment in the city of Bristol, UK. The test-bed experiments also consider multi-hop topologies. Lastly, this paper evaluates the robustness of the proposed scheme on temperature differences that severely affect the clock drifts [7]. In particular, adaptive guard time is experimentally evaluated in the SPHERE house test-bed [8] in links with 
temperature difference (indoors-to-outdoors). Overall, this paper extends our preliminary works with extensive experimentation, and hence, strengthens and generalises our findings in a wide range of realistic scenarios.

\section{Background and Related Work}

TSCH is among the MAC protocols defined in the IEEE 802.15.4-2015 standard, and promises performance levels that are competitive to industrial standards, such as ISA [11], WirelessHART [1]. This section provides the necessary background information about the mechanics of TSCH and reviews the related work.

\subsection{IEEE802.15.4-2015 TSCH}

TSCH achieves high level of network reliability and low-power operation by combining channel hopping and time synchronisation. Packet transmissions are organised by a deterministic scheduling scheme and the continuous time is divided into timeslots of equal size, while a set of timeslots form a slotframe which continuously repeats over time. At each timeslot, a node may transmit a packet and receive an acknowledgement. The timeslots are either contention-free (i.e., dedicated) or contention-based (i.e., shared). Finally, each timeslot comes with Absolute Slot Number (ASN) variable which indicates the number of timeslots since the beginning of the TSCH network.

Typically the clocks of the devices drift relatively their neighbours' due to production spread, as well as temperature changes. In TSCH, a node may resynchronise to its time source neighbour according to either a frame-based or acknowledgement-based scheme [20]. To re-synchronise, the receiver node computes the offset, i.e. the relative de-synchronisation time. Then, under framebased synchronisation, the offset is handled at the receiver side, while in ACKbased, it is applied to the transmitter's timing. The nodes regularly transmit Enhanced Beacon (EB) packets to re-synchronise their clocks and, consequently, to remain time-synchronised during the network's lifetime. In addition, data packets may also be employed to calculate the clock drifts [5]. EB packets contain the channel hopping sequence, the current ASN and information related to the initial link and slotframe. Finally, by receiving the EB packets, new nodes may join an existing TSCH network.

Each TSCH receiver activates its radio before the scheduled transmission by a fixed amount of time to avoid the loss of synchronisation between the synchronisation events. This guard time controls a performance trade-off. A longer guard time yields better resilience to loss of synchronisation, and therefore higher PDR. Yet, this comes at the cost of higher energy consumption, as more energy is lost in idle listening. 


\subsection{Related Work}

Previous works improve the energy efficiency of TSCH networks either by improving the scheduler $[14,16]$ or the synchronisation process. In this section, we briefly discuss the latter.

In [19], the authors present an adaptive synchronisation technique, and suggest that in a TSCH network the resynchronisation frequency should be reduced while the nodes are synchronised. In order to keep synchronisation, the nodes measure their clock drift rates relative to their time source neighbours. Later, the nodes periodically perform a time correction based on the previously calculated offset. As a result, this frequency reduction technique allows for lower duty cycle and longer network lifetime. In [4], the authors propose an adaptive compensation method that enables synchronisation between nodes, without exchanging any control or data packet. To this aim, the proposed algorithm compensates the clock drift error by regularly adjusting the interval of resynchronisation (i.e., local clock) of each node to improve the accuracy of the clock drift.

In [5], the authors propose an adaptive synchronisation scheme which allows the nodes to learn and predict how their clocks are drifting relative to their neighbours. Later, they coordinate the instants at which the nodes will re-synchronize. To this aim, an additional field in the EB and ACK packets is introduced to inform the neighbour node about the synchronised time. The authors evaluated the proposed technique in a TSCH multi-hop topology. In [9], the authors improve the level of synchronisation of TSCH networks by one order of magnitude in comparison to [5]. This is achieved by improving the resolution of measurement of the drift via keeping the high- and low-frequency clocks of a sensor node synchronised. This high level of synchronisation allows the reduction of the guard time and, therefore, the reduction of the energy consumption. In [7], the authors extend this algorithm with temperature calibration and provide an solution for temperature-resilient time synchronisation. In [12], the authors present a detailed analysis of the main factors contributing to the energy-consumption of Wireless HART standard, a Time Division Multiple Access (TDMA)-based and frequency hopping protocol [1]. Similar to TSCH protocol, under Wireless HART, the nodes listen the medium for certain time before the reception of control or data packets. The authors demonstrated that in addition to the radio transmission and reception states, the listening state in data and control timeslots has a significant impact of the energy consumption of a node.

Directly or indirectly, the above works improve the energy efficiency of TSCH by enhancing the process of synchronisation. Indeed, these works demonstrate that significant energy savings can be achieved through tighter time synchronisation. Yet, all the above works keep the guard time static throughout the network. Different to the related work, this paper proposes the adaptation of the guard time at a node level in a fully distributed fashion. This technique exploits the individual properties of each node, such as the distance to the sink. Indeed, adaptive guard times can be used together with adaptive synchronisation techniques for combined benefits. 


\section{Guard Time Adaptation}

As the number of hops increases, the aggregated relative clock drift increases as well [9]. Therefore, multi-hop TSCH networks require longer guard times to maintain their synchronous operation. By employing the default version of TSCH in a multi-hop network, all nodes homogeneously operate with a static guard time configuration (i.e., $2200 \mu$ s for CC2420, $1800 \mu$ s CC2650) [6]. Indeed, the nodes that are closer to the sink forward more packets and, thus, synchronise more frequently than leaf nodes. Therefore, the nodes that are closer to the sink require shorter guard times to maintain synchronisation.

This paper proposes a fully-distributed guard time adaptation algorithm for improving the energy efficiency of TSCH without compromising its reliability. Following a heuristic approach, each node adapts its guard time according to its runtime hop-distance to the time source (sink node). The runtime hop-distance is defined as the number of hops required to reach the sink node, according to the respective topology of the routing layer. In particular, the proposed scheme operates as follows. During an off-line calibration phase, the scheme constructs an empirical look-up table of optimum guard times, according to the number of hops from the root. At the end of the calibration process, shown in Algorithm 1, the look-up table contains the minimum guard times that do not break time synchronisation. At runtime, once the RPL [21] Direction-Oriented Directed Acyclic Graph (DODAG) is successfully constructed and, thus, each node in the network obtain all the necessary information related with its rank, each node adjusts its guard time according to the look-up table, as shown in Algorithm 2. It is straightforward that the nodes closer to the DODAG root will maintain shorter guard times, since they frequently participate in the routing procedure and, thus, they constantly re-synchronise their clocks, while the leaf nodes will have longer guard times. Finally, the nodes reconfigure their guard time in a decentralised and local manner, and the runtime overhead is minimal, i.e., $O(1)$.

In this paper, we propose guard time adaptation according to the hopdistance to the sink. This technique can be combined with other guard time adaptation techniques that are based on other parameters that affect synchronisation, such as the temperature [7].

\section{Performance evaluation}

In order to evaluate the benefits of guard time adaptation, as well as the impact of the guard time in the energy consumption of a TSCH network, a series of simulations are conducted in COOJA, the network simulator distributed as part of the Contiki OS, emulating the CC2420-based Z1 motes. In addition, a series of test-bed experiments are executed in the SPHERE house test-bed [8], a deployment of CC2650-based sensor nodes [10] in a real residential environment in the city of Bristol, UK. This section presents a thorough performance evaluation for both statically configured guard times and the proposed adaptive guard time

scheme. The details of the simulation and test-bed setup are given in Table 1, unless explicitly stated otherwise. 

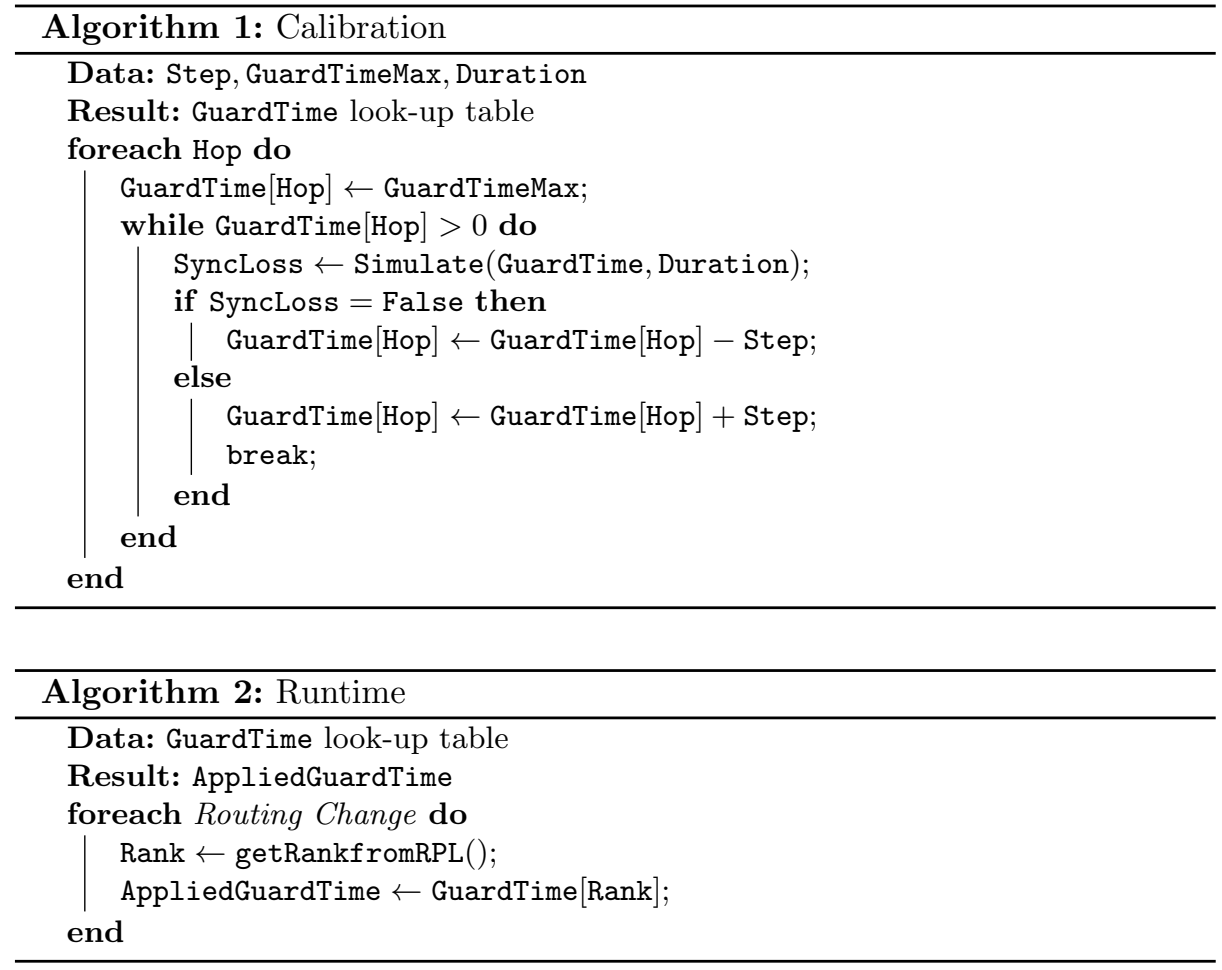

\subsection{Simulation: Multi-Hop Line Topologies}

The next series of simulations evaluate guard time adaptation in a 9-hop multihop chain topology, shown in Figure 1. The traffic rate of each node is set to one packet per minute. The simulations focus on a worst case scenario for time synchronisation: odd nodes have positive clock drift and even nodes have negative clock drift. Moreover, the nodes use the 6 TiSCH minimal schedule. Finally, the duration of each simulation is $60 \mathrm{~m}$.

The goal of the first set of simulations is to identify the minimum guard time that yields $100 \%$ PDR in each layer of the network. The empirically-derived minimum guard time for each hop is illustrated in Figure 1 and used as a lookup table for the implementation of guard time adaptation in Contiki. The results verify that nodes closer to the sink require a smaller guard time as opposed to the leaf nodes. The empirically-derived network-wide optimum guard time is $1200 \mu \mathrm{s}$. Therefore, this static configuration is used as a benchmark for the evaluation of the proposed adaptive guard time scheme.

The following simulations quantify the benefits of adapting the guard time according to the hop-distance to the sink. A set of simulations evaluates the proposed scheme in various application-layer traffic rates, focusing on the 9-hop line topology. Figure 2 shows that the adaptation of the guard time improves the energy efficiency of the TSCH network, by reducing the radio duty cycle. This 
Adaptive Guard Time for Energy-Efficient IEEE 802.15.4 TSCH Networks

Table 1. Simulation \& Experimental Setup

\begin{tabular}{|c|c|c|}
\hline & Simulation & Experiments \\
\hline \multicolumn{3}{|l|}{ Multi-Hop Line Topology } \\
\hline Number of Devices & From 2 to 10 & 10 \\
\hline Node spacing & $100 \mathrm{~m}$ & $2 m-6 m$ \\
\hline \multicolumn{3}{|l|}{ Multi-Hop Random Topology } \\
\hline Number of Devices & 15 & - \\
\hline Node spacing & random & - \\
\hline Maximum Number of Hops & 6 & - \\
\hline \multicolumn{3}{|l|}{ Indoor-to-Outdoor Link } \\
\hline Number of Devices & - & 2 \\
\hline Node spacing & - & $2 \mathrm{~m}$ \\
\hline \multicolumn{3}{|c|}{ Duration, Network and Traffic parameters } \\
\hline Duration & 1 hour & $\begin{array}{l}\text { 1-hop: } 24 \text { hours } \\
\text { Multi-hop: } 1 \text { hour }\end{array}$ \\
\hline Traffic Rate & $1-512 \mathrm{pkt} / 60 \mathrm{sec}$ & $1 \mathrm{pkt} / 60 \mathrm{sec}$ \\
\hline Packet size & 102 bytes & 102 bytes \\
\hline Routing & $\mathrm{RPL}[21]$ & $\mathrm{RPL}[21]$ \\
\hline $\mathrm{MAC}$ & $\mathrm{TSCH}$ & $\mathrm{TSCH}$ \\
\hline Schedule & 6TiSCH minimal & Collision-free \\
\hline \multicolumn{3}{|l|}{ TSCH-specific parameters } \\
\hline EB frequency & $3.42 \mathrm{~s}$ & $4 \mathrm{~s}$ \\
\hline Slotframe length & 7 & 17 \\
\hline Timeslot length & $15 \mathrm{~ms}$ & $10 \mathrm{~ms}$ \\
\hline Guard Time & $(0-2200) \mu \mathrm{s}$ & $(0-1600) \mu \mathrm{s}$ \\
\hline Clock Drift & $\pm 20 \mathrm{ppm}$ & $\begin{array}{c}\text { Natural drift } \\
\text { up to } \pm 20 \mathrm{ppm}\end{array}$ \\
\hline \multicolumn{3}{|l|}{ Hardware configuration } \\
\hline Radio hardware & $\mathrm{CC} 2420$ & CC2650 \\
\hline Radio frequency & $2.4 \mathrm{GHz}$ & $2.4 \mathrm{GHz}$ \\
\hline TX power & $0 \mathrm{dBm}$ & $5 \mathrm{dBm}$ \\
\hline
\end{tabular}

reduction is more significant (i.e., up to 50\%) for TSCH networks that run lowthroughput applications and drops as the traffic increases. This happens because in high traffic scenarios the radio is primarily used for transmissions.

\subsection{Simulation: Multi-Hop Random Topologies}

The following simulations evaluate the proposed algorithm in multi-hop random topologies of 15 nodes to further validate its efficiency in realistic TSCH networks. Similarly to the previous simulations the clock drift is fixed to $\pm 20 \mathrm{ppm}$, while 1 data packet was transmitted per minute. The simulations demonstrate that the guard time of the receiver nodes can be reduced without compromising the reliability of the network (100\% PDR). Indeed, as depicted in Figure 3, the average radio duty cycle is reduced by approximately $20 \%$. Moreover, the con- 


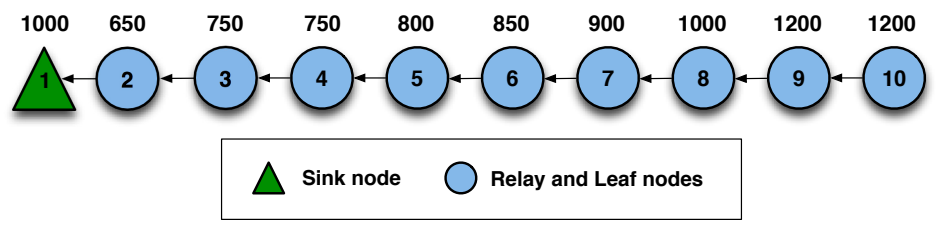

Fig. 1. The simulated line topology. The number above each node corresponds to the minimum configuration that maintains $100 \%$ network reliability (in $\mu \mathrm{s}$ ).

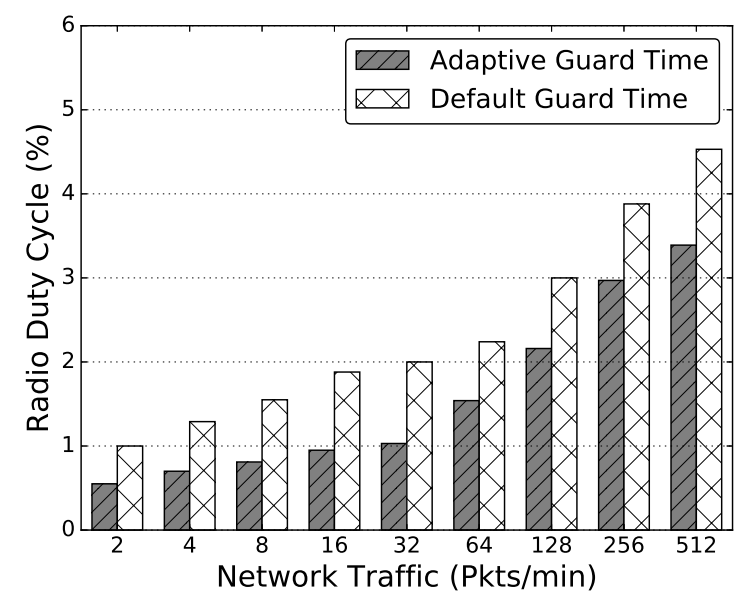

Fig. 2. Average radio duty cycle for various application-layer traffic rates.

sistency of the results in 10 random networks demonstrate the robustness of the proposed algorithm to the network topology.

\subsection{Experiment: Multi-Hop Line Topology}

Next, guard time adaptation is evaluated in experiments, conducted in the SPHERE house test-bed [8]. The SPHERE house test-bed is a wireless sensor network deployed in a 2-storey residential environment in the city of Bristol, UK, shown in Figure 4. In particular, the house is deployed with TI CC2650based sensor nodes [10] (Figure 5); a platform that is supported by the Contiki OS. All of the deployed nodes are equipped with the FC-135 low-frequency crystal, using it to schedule the wake-up events. This crystal has a frequency error $e_{f} \in[-20,20] \mathrm{ppm}$ and a parabolic dependence on temperature (parabolic coefficient value $B=0.04 \mathrm{ppm} /{ }^{\circ} \mathrm{C}^{2}$ ). The channel conditions in the SPHERE house are typical for an urban residential neighbourhood, with occasional $2.4 \mathrm{GHz}$ interference from the neighbouring houses. The nodes are sufficiently close to form 


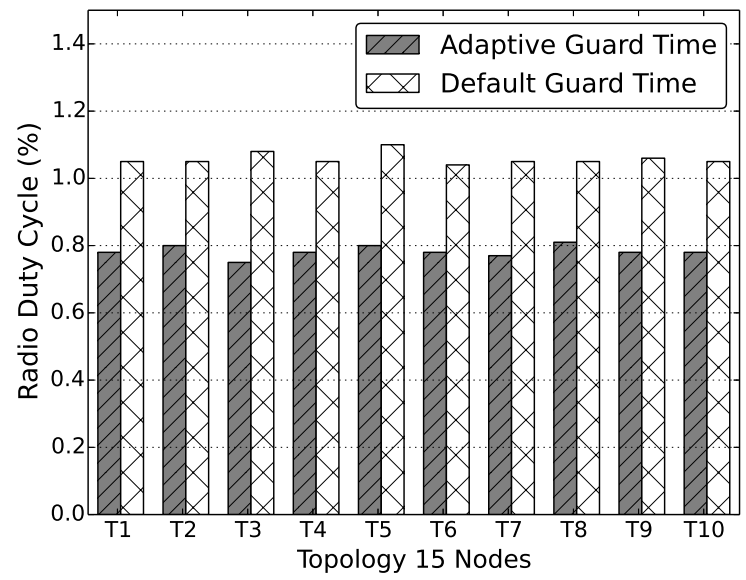

Fig. 3. Guard time adaptation evaluated in 10 random multi-hop topologies of 15 nodes

a single-hop star topology. Yet, for the purposes of this experiment, the nodes use a static schedule that enforces a 9-hop line topology, similar to Figure 1.

In this setting, the proposed adaptive guard time scheme is compared against the Contiki default fixed guard time in two 1-hour experiments. Fig. 7 summarises the experimental results, which show the reduction of the radio duty cycle in each of the 10 nodes in the test-bed. The experiments verify the simulations presented in Section 4.1, indicating an average 15\% reduction in the radio duty cycle without sacrificing the reliability (100\% PDR). The experiments show that nodes that are closer to the sink (i.e., shorter hop-distance) relay more traffic, and hence, synchronise with their parent node more frequently. Thus, a smaller guard time is sufficient and energy can be saved by adapting it.

\subsection{Experiment: Indoors-to-Outdoors}

Clock drifts are also dependent on temperature [7]. This section presents an experiment that considers a link with temperature differences, i.e., the sender is indoors $\left(22{ }^{\circ} \mathrm{C}\right.$ room temperature) while the receiver is outdoors and, therefore, exposed to outdoor temperatures. The proposed adaptive guard time algorithm is, again, compared against the default configuration in 24-hour experiments. Fig. 6 summarises the results of the experiment. In particular, Fig. 6a plots the radio duty cycle over the course of the 24-hour experiment. Both approaches demonstrate a fairly constant duty cycle, yet present a periodicity that is attributed to the temperature variations, shown in Fig. 6b. Despite these temperature variations, both configurations operate robustly in this 24-hour experiment, yielding 100\% PDR. Yet adaptive guard time reduces the radio duty cycle, and thus, the energy consumption of the radio by approximately $25 \%$. 


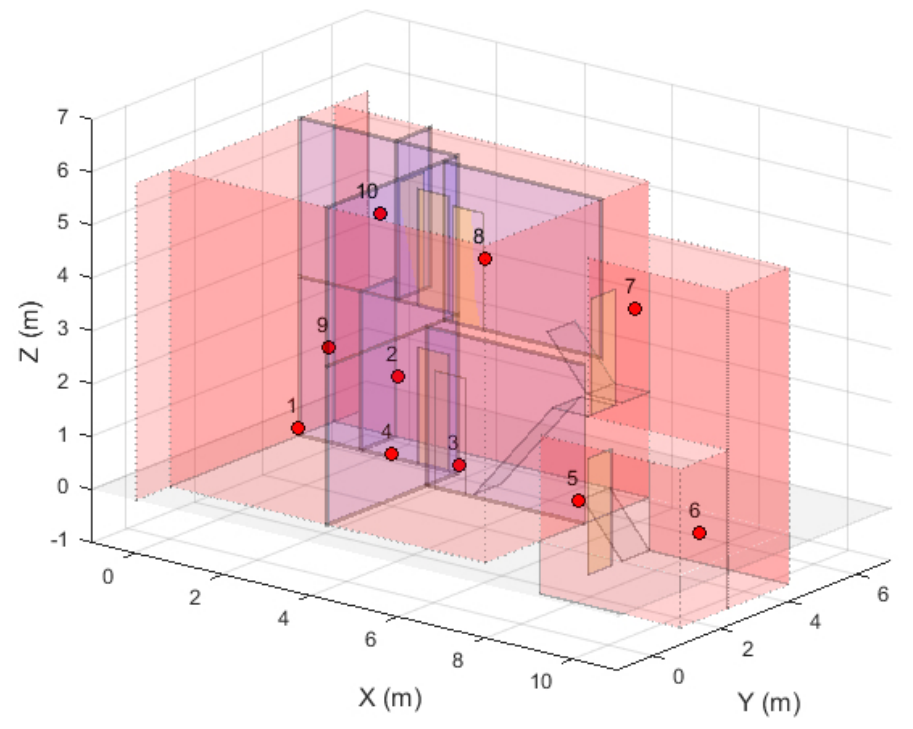

Fig. 4. The SPHERE house test-bed is a deployment of C2650-based nodes in a twostorey residential environment in Bristol, UK [8].
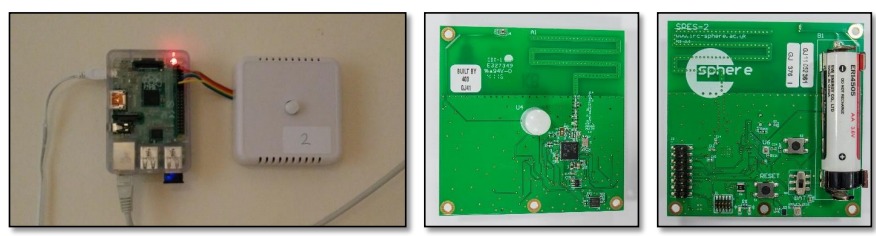

Fig. 5. Experiments were conducted on the SPES-2 [10] sensing platform; a environmental sensor that is equipped with the TI CC2650 radio.

\section{Conclusions}

This paper investigates the impact of guard time on IEEE 802.15.4 TSCH networks and proposes a decentralised guard time adaptation algorithm for reducing the energy consumption of TSCH-based multi-hop networks. The proposed technique follows the formation of the network topology and allows each node to assign its own guard time according to its distance in hops from the sink node. This dynamic procedure allows the network nodes to reduce the time they spend in idle listening; therefore, the energy consumption is significantly reduced. The proposed algorithm is implemented in Contiki OS and evaluated with an extensive set of simulations, which include several types of topologies, such as multi-hop line and random topologies. In addition, the proposed algorithm is also evaluated on the SPHERE test-bed. The results of the simulations and test-bed experiments demonstrate the advantage of adapting the guard time instead of using a static configuration. 


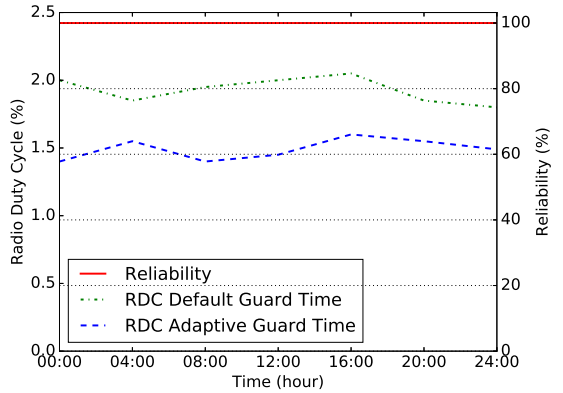

(a)

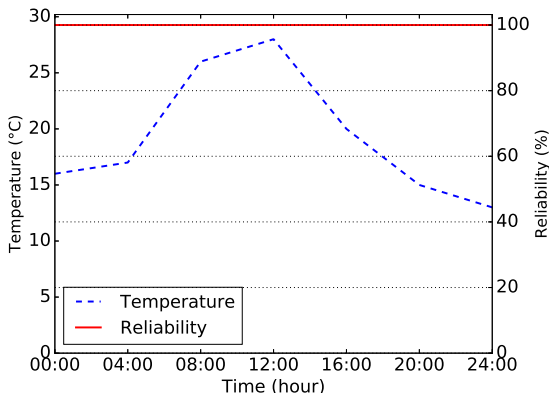

(b)

Fig. 6. Adaptive guard times reduce the average radio duty cycle without compromising the reliability (a) despite the temperature differences (b). In this 24-hour experiment, the sender is indoors (room temperature) whilst the receiver is exposed to various outdoor temperatures.

\section{References}

1. WirelessHART Specification 75: TDMA Data-Link Layer. Std., Rev 1 (2008)

2. IEEE Standard for Low-Rate Wireless Personal Area Networks (LR-WPANs). IEEE Std 802.15.4-2015 (Revision of IEEE Std 802.15.4-2011) (April 2016)

3. Brzozowski, M., Salomon, H., Langendoerfer, P.: On efficient clock drift prediction means and their applicability to ieee 802.15.4. In: 2010 IEEE/IFIP International Conference on Embedded and Ubiquitous Computing. pp. 216-223 (Dec 2010)

4. Chang, T., Wang, Q.: Adaptive compensation for time-slotted synchronization in wireless sensor network. International Journal of Distributed Sensor Networks 2014(9) (2014)

5. Chang, T., Watteyne, T., Pister, K., Wang, Q.: Adaptive Synchronization in Multihop TSCH Networks. Computer Networks 76, 165-176 (2015)

6. Duquennoy, S., Elsts, A., Nahas, B.A., Oikonomou, G.: TSCH and 6TiSCH for Contiki: Challenges, Design and Evaluation. In: 13th Int. Conf. on Distributed Comput. in Sensor Syst. (DCOSS) (2017)

7. Elsts, A., Fafoutis, X., Duquennoy, S., Oikonomou, G., Piechocki, R., Craddock, I.: Temperature-resilient time synchronization for the internet of things. IEEE Transactions on Industrial Informatics 14(5), 2241-2250 (May 2018)

8. Elsts, A., Fafoutis, X., Woznowski, P., Tonkin, E., Oikonomou, G., Piechocki, R., Craddock, I.: Enabling Healthcare in Smart Homes: The SPHERE IoT Network Infrastructure. IEEE Communications Magazine 56(12), 164-170 (2018)

9. Elsts, A., Duquennoy, S., Fafoutis, X., Oikonomou, G., Piechocki, R., Craddock, I.: Microsecond-Accuracy Time Synchronization Using the IEEE 802.15.4 TSCH Protocol. In: IEEE SenseApp (2016)

10. Fafoutis, X., Elsts, A., Vafeas, A., Oikonomou, G., Piechocki, R.: Demo: SPES-2 A sensing platform for maintenance-free residential monitoring. In: EWSN (2017)

11. ISA-100.11a-2011:: Wireless systems for industrial automation:process control and related applications. International Society of Automation (ISA) Std. 1 (May 2011)

12. Khader, O., Willig, A.: An Energy Consumption Analysis of the Wireless HART TDMA Protocol. Computer Communications (2013) 


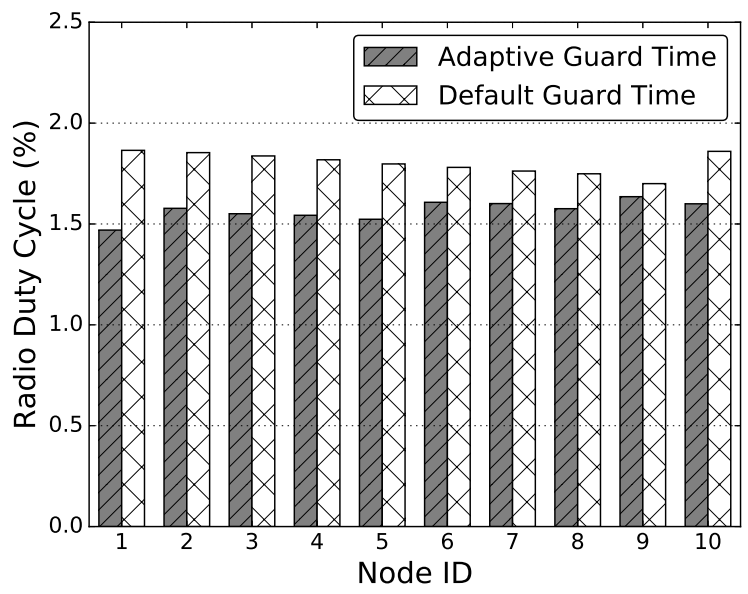

Fig. 7. The practical benefits of guard time adaptation in the SPHERE house test-bed (9-hop line topology). The PDR is $100 \%$ in all the experiments.

13. Koutsiamanis, R.A., Papadopoulos, G.Z., Fafoutis, X., Fiore, J.M.D., Thubert, P., Montavont, N.: From Best Effort to Deterministic Packet Delivery for Wireless Industrial IoT Networks. IEEE Transactions on Industrial Informatics 14(10), 4468-4480 (2018)

14. Matsui, T., Nishi, H.: Time slotted channel hopping scheduling based on the energy consumption of wireless sensor networks. In: 2018 IEEE 15th International Workshop on Advanced Motion Control (AMC). pp. 605-610 (March 2018)

15. Mavromatis, A., Papadopoulos, G.Z., Fafoutis, X., Elsts, A., Oikonomou, G., Tryfonas, T.: Impact of Guard Time Length on IEEE 802.15.4e TSCH Energy Consumption. In: Proceedings of the IEEE SECON (2016)

16. Ojo, M., Giordano, S., Portaluri, G., Adami, D., Pagano, M.: An energy efficient centralized scheduling scheme in tsch networks. In: 2017 IEEE International Conference on Communications Workshops (ICC Workshops). pp. 570-575 (May 2017)

17. Papadopoulos, G.Z., Mavromatis, A., Fafoutis, X., Montavont, N., Piechocki, R., Tryfonas, T., Oikonomou, G.: Guard Time Optimisation and Adaptation for Energy Efficient Multi-hop TSCH Networks. In: IEEE WF-IoT. pp. 301-306 (2016)

18. Papadopoulos, G.Z., et al.: Guard Time Optimisation for Energy Efficiency in IEEE 802.15.4-2015 TSCH Links. In: Proceedings of the EAI International Conference on Interoperability in IoT (InterIoT) (2016)

19. Stanislowski, D., Vilajosana, X., Wang, Q., Watteyne, T., Pister, K.S.J.: Adaptive Synchronization in IEEE802.15.4e Networks. IEEE Transactions on Industrial Informatics 10(1), 795-802 (2014)

20. Thubert, P.: An Architecture for IPv6 over the TSCH mode of IEEE 802.15.4. draft-ietf-6tisch-architecture-20 (March 2019)

21. Winter, T., Thubert, P., Brandt, A., Hui, J., Kelsey, R., Levis, P., Pister, K., Struik, R., Vasseur, J., R., A.: RPL: IPv6 Routing Protocol for Low-Power and Lossy Networks. RFC 6550 (2012)

22. Xu, L.D., He, W., Li, S.: Internet of things in industries: A survey. IEEE Transactions on Industrial Informatics 10(4), 2233-2243 (Nov 2014) 